\title{
A Broad Handbook for Partnering With Stakeholders in Translational Health Research
}

\author{
Rebecca E Rosenberg ${ }^{1}$ (D) \\ ${ }^{1}$ Department of Pediatrics, NYU Grossman School of Medicine; Hassenfeld Children's Hospital at NYU Langone \\ Keywords: patient powered research, community engaged research, community based participartory research \\ 10.35844/001c.18391
}

Journal of Participatory Research Methods

\author{
Researching Health Together: Engaging Patients and \\ Stakeholders, From Topic Identification to Policy Change. Emily \\ B. Zimmerman, Ed. Sage Publications, Thousand Oaks, \\ California, 2021, $1^{\text {st }}$ ed.
}

This compendium of stakeholder-engaged research builds on a unifying premise: that developing, conducting, and promoting research in partnership with patients and affected communities is the ideal path for health research and the roadmap is heterogenous. Like other graduate-level research methods handbooks, the chapters, often cowritten with academic and community investigators, each showcase an exemplar of research with varying degrees of practical details.

This book differs from community-based participatory research (CBPR) texts which can be generalized to any type of sociologic research. The scope of "Researching Health Together" focuses only on translational health research and expands beyond CBPR to include practice-based research networks (PBRN) and stakeholder-engaged research within health systems. Its contributors have backgrounds in nonprofit leadership, community organizing, social science and policy, and translational research from North America, the United Kingdom, and Native communities. The breadth of health-focused research in various settings, including health systems, makes this handbook relevant and accessible to health researchers with little exposure or knowledge of doing research with, rather than only on or for, communities.

As the title promises, chapters consist of exemplar descriptions of successful, often longstanding, participatory health research grouped into sections, including: research topic identification; research design; and implementation, as well as sections on health systems interventions and governance, evaluation, and ethics. As a textbook, there are discussion questions at the end of each chapter, which vary in type and focus, as well as section overviews. The bulk of the chapters/examples hew more toward CBPR but do include some of the finest and most well-known PBRN projects, including the High Plains Research Network and its Boot Camp Translation methodology. Most projects reported funding from local and/or regional grants as well as the Patient Centered Outcomes Research Institute (PCORI) or another federal funder. In other words, this volume is not aimed at the casual reader with 
limited community engagement resources but rather highlights aspirational engagement and offers diverse ideas of participatory research methods in translational health research.

The Introduction (Zimmerman and Concannon) is notable for both its clarity and comprehensiveness in summarizing the field of participatory health research including a typology of stakeholder engagement approaches in research. The Introduction offers an important scoping summary of the field. The Glossary, too, is useful for ensuring that readers are using a common vocabulary in a relatively newer field with much de novo terminology. Together, these two assets invite and ground the novice participatory health researcher to explore this compilation of methodologies.

Like qualitative methodology, participatory health research necessarily highlights context. Each chapter first details a project or case, focusing and deeply describing methods highlighting a specific concept, such as implementation. Because no universal framework was used to structure the chapters, comparing various projects could be difficult for the novice reader. On the other hand, the wide variety lends the reader many ideas.

The overall strengths of this book are its in-depth and almost inspirational focus on CBPR methodology, be those actual geographic or cultural communities or disease-based communities. PBRN are also well represented. There is admittedly little on much smaller projects or how to engage with stakeholders outside of large, resourced efforts.

Common themes across the chapters include the large investment of time and resources needed to properly engage stakeholders and then maintain their engagement. Some studies did mention compensation to community stakeholders, which demonstrates face-validity in terms of parity on contributions between community member and researchers. The chapters on ethics touch on the larger issues, especially for community participants who can literally have two roles of being both community member but also research team member and representative. Surprisingly, the role and importance of participatory health research to address health disparities and target health equity is inferred, but not discussed as primary aim. Another key theme was setting expectations for communities on deliverables. For example, several projects described how stakeholders were disappointed in the end products, such as research agendas, when they may have preferred concrete interventions to improve health in their community.

What is striking in the field of participatory health research as aggregated in this handbook is the consistency of methodologic principles rather than exact methodology. The editors and contributors offer de novo schema and even less formal "lessons learned," which suggest that the organic nature of partnership as well as the field's real-time evolution make this a first, but certainly not last, edition. Concannon's later chapter, "Evaluating Engagement," is an important ballast to the excitement of the rest of the volume. As in qualitative research, there remains some missing fidelity and implementation questions as to how 
robust a partnership is to fall on the side of meaningful versus tokenistic. At the same time, not every project can support heavy participation and engagement of stakeholders given the resources needed to do it well.

"Researching Health Together," in its first edition, is a necessary bridge from the theory of participatory health research to its application across research environments.

Submitted: October 23, 2020 EST, Accepted: December 07, 2020 EST

This is an open-access article distributed under the terms of the Creative Commons Attribution 4.0 International License (CCBY-4.0). View this license's legal deed at http://creativecommons.org/licenses/ by/4.0 and legal code at http://creativecommons.org/licenses/by/4.0/legalcode for more information. 